\title{
Effects of laser fusing of colour ceramic layers with glass surface during front-side and back-side glass irradiation
}

\author{
A. Sarzyński ${ }^{* 1}$, D. Chmielewska ${ }^{2}$ J. Marczak ${ }^{1}$, A. Olszyna ${ }^{2}$, M. Strzelec ${ }^{1}$, R. Gebel ${ }^{2}$ \\ ${ }^{1}$ Institute of Optoelectronics, Military University of Technology, 2 Gen. Sylwestra Kaliskiego Street, 00-908 \\ Warszawa, Poland, \\ ${ }^{2}$ Institute of Ceramics and Building Materials, 9 Postępu Street, 02-676 Warszawa, Poland
}

Received August 26, 2015; accepted September 28, 2015; published September 30, 2015

\begin{abstract}
By reducing the costs and time of a production process and by introducing significant improvements in technological processes, laser fusing of colour ceramic agents with ceramic or glass products arouses great interest, thereby substituting the typical process of furnace firing in decorating small series of products. The glass substrate of a decoration allows for using two configurations of an optical scheme in the technological process - laser irradiation from the side of a fused ceramic layer as well as from the side of a glass substrate. The presented results are mainly related to experimental determination of utility features and advantages of both solutions.
\end{abstract}

The laser technique has been more and more widely applied to decorate ceramic and glass products for more than 10 years $[1,2]$. Laser fusing of ceramic colour agents with the decorated substrate of a product [3], exhibiting high durability, temperature and chemical resistance is an increasingly used method.

Physico-chemical properties of decorative, hardened glass vary considerably compared to a typical substrate of most ceramic products. Primarily, lower softening temperatures, differences in short-time heat conduction, and volumetric reaction on temporary temperature gradients, created by the thermal interaction of a laser beam, are important for the laser process. Moreover, unlike the laser decoration of opaque ceramic products [4], glass substrates allow laser fusing of colour decorative agents both directly - from the front side of product, as well as throughout the glass layer. Both configurations are characterised by large differences in optimal parameters of technological processes and as a result, decoration quality, which was demonstrated in a series of experiments described below.

The processes were researched and optimised using computer-controlled ytterbium-doped diode-pumped fibre laser systems: GLPM 10 from IPG Inc, USA (10W, $625 \mathrm{kHz}$ repetition rate, $1 \mathrm{~ns}$ pulse duration, $532 \mathrm{~nm})$ and SP-100C-0020 from SPI Lasers UK Ltd (CW, 100W, $1070 \mathrm{~nm})$, coupled with "galvo" type beam scanners RLC1004 from Raylase GmbH, Germany.

*E-mail: antoni.sarzynski@wat.edu.pl
Tests were performed with an extensive range of colour agents developed at the Institute of Ceramics and Building Materials in Warsaw, and with commercial glass substrates, delivered by an industrial project partner Ceramika Tubądzin II Sp. z o.o.

In the case of laser radiation interacting directly with the colour material, fusing with a substrate takes place through the layer of ceramic paint. Therefore, the process is dependent on the thickness of a coating material. Coating thickness determines also energy doses (laser fluence), optimal from the point of decoration quality: smoothness, specified by roughness parameters, homogeneous paint distribution in composition, and the quantity and size of defects - coating losses and cracks in the layer itself or in the glass substrate.
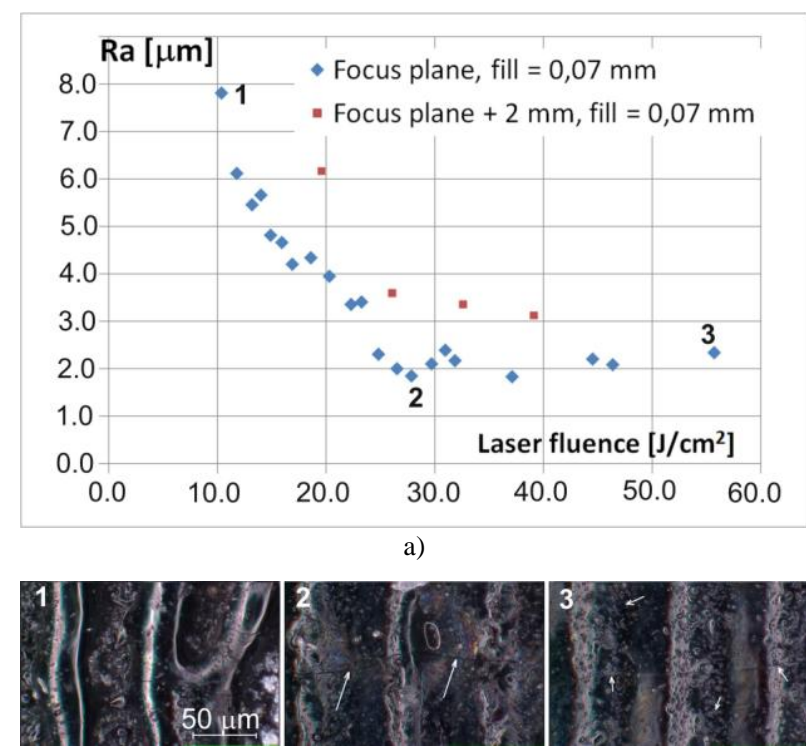

b)

Fig. 1. a) Dependence of roughness parameter Ra of fused paint on laser fluence; b) Microphotographs of paint surface in the points indicated on plot a). Black ceramic paint with technological symbol 6FS1500CZ, fibre laser SPI (1070nm), fill - distance between adjacent scanning lines, two indicated locations of laser processing planes. 
Figure 1a shows changes of roughness parameter $\mathrm{Ra}$ depending on a dose of irradiation laser energy (laser fluence). Three characteristic points, indicated on the plot (Fig. 1a) are additionally illustrated by their optical microphotographs (Fig. 1b). Point 1 (high Ra) is characterised by the flow of a fused colour agent to the edges of the scanning line [5] and losses in the decoration surface. Point 2 (minimal Ra) shows the levelling of fused paint and formation of initial microcracks (indicated by white arrows in the microphotograph). Point 3 illustrates an increase in the quantity and width (size) of microcracks leading to a slight increase of the Ra parameter.

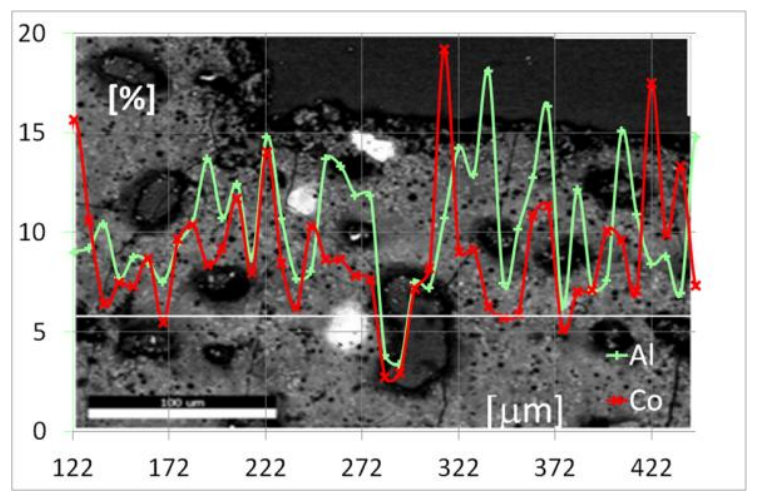

Fig. 2. Linear distribution of basic pigment components $(\mathrm{Al}, \mathrm{Co})$ in blue paint with technological symbol 6FS1664N along white line imposed on a SEM image of decoration fragment near the border of glass substrate. IPG laser $(532 \mathrm{~nm})$, laser fluence $28 \mathrm{~J} / \mathrm{cm}^{2}$.

The irregularity of surface distribution of a colour agent has been shown on the example of pigment elemental distribution in blue paint with the technological symbol 6FS1664N (Fig. 2). This distribution, registered in a SEM EDS spectrum along the white line indicated in Fig. 2 has been superimposed with a corresponding SEM image. Distinct pigment grains and surface losses down to a glass substrate can be observed along with visible irregular distribution of pigment components.

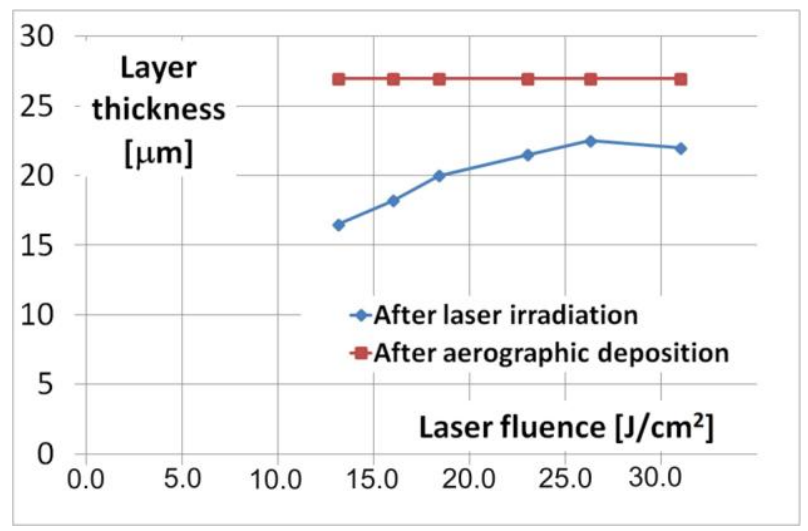

Fig. 3. Dependence of decoration layer thickness on laser radiation fluence. Green paint with technological symbol $2 \mathrm{~F}-\mathrm{Z} 4$.
In the second configuration, the thickness of the ceramic layer, fused by irradiation through the glass layer does not depend on the initial thickness of aerographically deposited paint (Fig. 3). This feature has an important influence on the development of the target technology of laser decoration of glass. It allows assessing that thin and homogeneous aerographic coating of products before laser irradiation is not an important factor in this configuration.

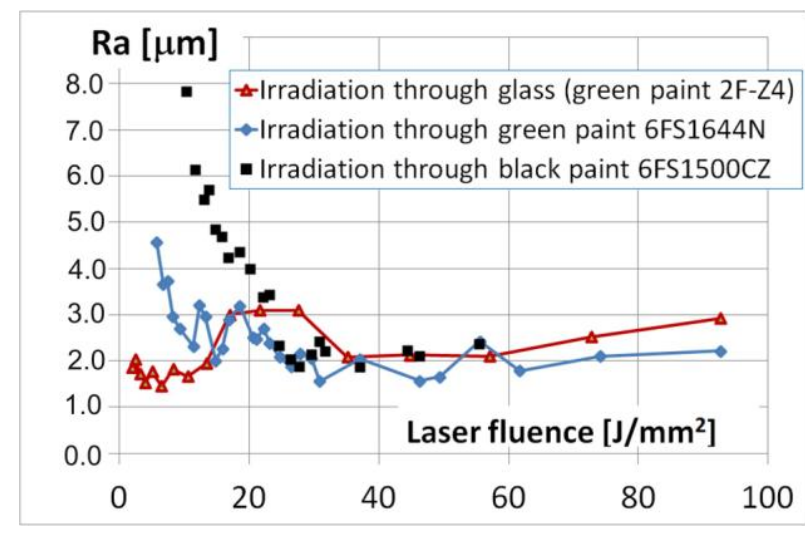

Fig. 4. Dependence of Ra roughness parameter on laser fluence and configuration of laser irradiation.

The second advantage of sample irradiation through the glass layer is the low value of roughness parameters for low values of laser fluence, characteristic for minimisation of decoration cracks. Figure 4 illustrates a comparison of the dependence of roughness parameter $\mathrm{Ra}$ on a laser energy dose for both optical configurations discussed. In the summary of all tests conducted with both fibre lasers, definitely better results are obtained for the laser operating at a wavelength of $532 \mathrm{~nm}$ rather than $1070 \mathrm{~nm}$. It corresponds to higher absorption of colour agents in the visible part of the spectrum.

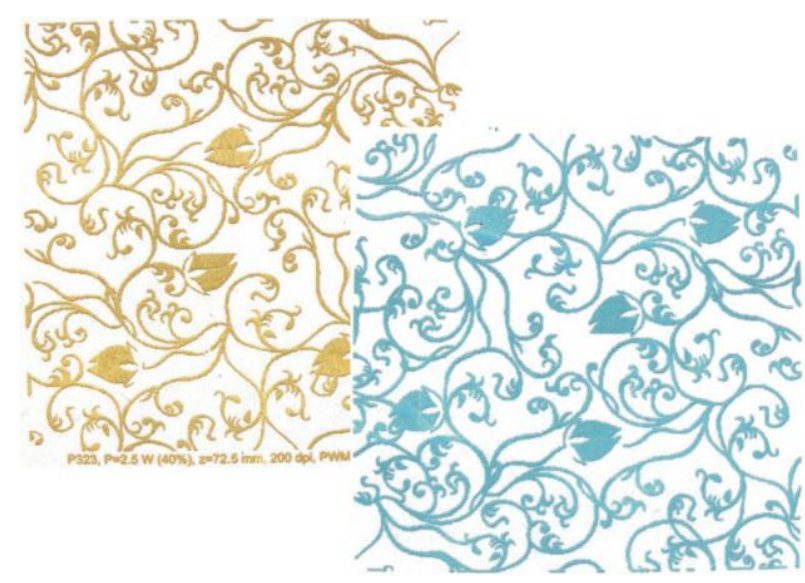

Fig. 5. Example of laser fused colour decoration showing image of vector ornament (Corel Draw). 
Irradiation through a glass layer assures lower optimal energy doses (laser fluence), and in consequence minimisation of defects (microcracks, surface losses), high smoothness of a fused layer and more homogeneous distribution of paint components, which in turn increases uniformity of colour saturation. The technology has been optimised both for vector graphics and raster bitmaps. Examples are shown in Fig. 5 and in Fig. 6.
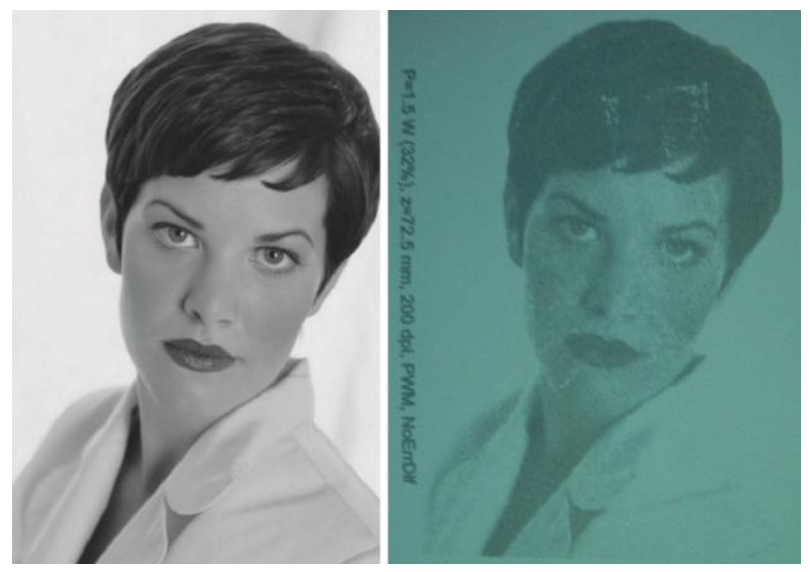

Fig. 6. Example of laser fused colour decoration showing a bitmap image (left side) and its laser projection before washing off the excess of green paint (right side).

This research, with participation of the industrial partner - Ceramika Tubądzin II Sp. z o.o. [Limited] was financially supported by project PBS1/A5/11/2012 "Innovative technology of colour, laser decoration of flat glass with ceramic agents" of the National Centre for Research and Development in Poland.

\section{References}

[1] J.M. Fernández-Pradas et al., Appl. Surf. Sci. 253, 7733 (2007).

[2] A. Moreno et al., Adv. Sci. Techn., 68, 165 (2010.).

[3] D. Chmielewska, R. Gebel, K. Szamałek, M. Strzelec, J. Marczak, Proc. SPIE, 8703, 87030I (2013).

[4] R. Akhter, L. Li, R.E. Edwards, A.W. Gale, Appl. Surf. Sci. 208-209, 453 (2003).

[5] D. Chmielewska et al., Physics Procedia 5/1, 407 (2010). 